\title{
On Local Fractional Continuous Wavelet Transform
}

\author{
Xiao-Jun Yang, ${ }^{1}$ Dumitru Baleanu, ${ }^{2,3,4}$ H. M. Srivastava, ${ }^{5}$ and J. A. Tenreiro Machado ${ }^{6}$ \\ ${ }^{1}$ Department of Mathematics and Mechanics, China University of Mining and Technology, Xuzhou, Jiangsu 221008, China \\ ${ }^{2}$ Department of Chemical and Materials Engineering, Faculty of Engineering, King Abdulaziz University, P.O. Box 80204, \\ Jeddah 21589, Saudi Arabia \\ ${ }^{3}$ Department of Mathematics and Computer Sciences, Faculty of Arts and Sciences, Cankaya University, 06530 Ankara, Turkey \\ ${ }^{4}$ Institute of Space Sciences, Magurele, 077125 Bucharest, Romania \\ ${ }^{5}$ Department of Mathematics and Statistics, University of Victoria, Victoria, BC, Canada V8W 3R4 \\ ${ }^{6}$ Department of Electrical Engineering, Institute of Engineering of Porto, Rua Dr. António Bernardino de Almeida 431, \\ 4200-072 Porto, Portugal
}

Correspondence should be addressed to Xiao-Jun Yang; dyangxiaojun@163.com

Received 25 October 2013; Accepted 10 November 2013

Academic Editor: Carlo Cattani

Copyright (c) 2013 Xiao-Jun Yang et al. This is an open access article distributed under the Creative Commons Attribution License, which permits unrestricted use, distribution, and reproduction in any medium, provided the original work is properly cited.

We introduce a new wavelet transform within the framework of the local fractional calculus. An illustrative example of local fractional wavelet transform is also presented.

\section{Introduction}

Wavelet transforms have been applied successfully in the areas of signals analysis, data compression, and sound processing (see, for details, [1-6] and the references cited therein). Although there is scaled and shifted versions of a mother wavelet, the daughter wavelets are structured as follows (see [3-5]):

$$
\varphi_{a, b}(t)=\frac{1}{a^{1 / 2}} \varphi\left(\frac{t-b}{a}\right)
$$

where $a$ is the dyadic dilation, $b$ is the dyadic position, and $a^{-1 / 2}$ is the normalization factor. The expression of a onedimensional wavelet transform for a given continuous signal $f(t)$ is given by

$$
W_{\varphi} f(a, b)=\int_{-\infty}^{\infty} f(t) \overline{\varphi_{a, b}(t)} d t
$$

and the reconstruction formula becomes

$$
f(x)=C_{\varphi} \iint_{-\infty}^{\infty} \frac{1}{a^{2}} W_{\varphi} f(a, b) \varphi_{a, b}(t) d a d b
$$

where

$$
C_{\varphi}=\int_{-\infty}^{\infty} \frac{|f(x)|^{2}}{|x|} d x .
$$

Recently, fractional wavelet transform, as a generalization of the classical wavelet transform, was proposed in [7]. The one-dimensional fractal wavelet transform of a continuous signal $f(t)$ has the following form:

$$
W_{\varphi} f(a, b)=\iint_{-\infty}^{\infty} B(x, t) f(t) \overline{\varphi_{a, b}(x)} d t d x,
$$

where $B(x, t)$ denotes a bulk optics kernel.

The reconstructing formula of the input is defined as given by the following expression:

$$
f(x)=\frac{1}{C_{\varphi}} \iint_{-\infty}^{\infty} \frac{1}{a^{3}} W_{\varphi} f(a, b) B(x, t) \varphi_{a, b}(t) d a d b d t d t .
$$

We notice that the fractional wavelet transforms was applied to image encryption [8], to the simultaneous spectral analysis in [9], and to the composite signals in [10, 11]. For other definition of fractional wavelet transform, see [12] and the references cited therein. 
Keeping in mind the study of the fractal signals (local fractional continuous signals), a new local fractional wavelet transform was developed in [13] based upon the local fractional Fourier transform [14] via local fractional calculus [1518]. In this paper, we investigate the local fractional Fourier transform to deal with the local fractional wavelet transforms by implementing the local fractional calculus.

The organization of the paper is as follows. Section 2 presents the concept of local fractional Fourier transform and wavelet. Section 3 discusses the derivation of the local fractional continuous wavelet transform. Section 4 studies the wave space and Section 5 present an illustrative example. Finally, Section 6 outlines the main conclusions of our present investigation.

\section{Local Fractional Fourier Transform and Wavelet}

Let $f(x)$ be local fractional continuous function, which is denoted as follows (see [18]):

$$
f(x) \in C_{\alpha}(-\infty, \infty) .
$$

The space of local fractional continuous functions $C_{p, \alpha}[a, b]$, under $p$-norm, is given by (see [13])

$$
\|f\|_{p, \alpha}=\left(\frac{1}{\Gamma(1+\alpha)} \int_{a}^{b}|f(x)|^{p}(d x)^{\alpha}\right)^{1 / p}, \quad \text { for } 1 \leq p<\infty,
$$

where the operator is local fractional operator.

The space $L_{p, \alpha}[\mathbb{R}]$ norm on $C_{p, \alpha}[\mathbb{R}]$ is defined by

$$
\|f\|_{p, \alpha}=:\left(\frac{1}{\Gamma(1+\alpha)} \int_{-\infty}^{\infty}|f(x)|^{p}(d x)^{\alpha}\right)^{1 / p}<\infty
$$

for $1 \leq p<\infty$. This is infinite for $a$ and $b$.

The local fractional Fourier transforms in fractal space is defined as follows (see $[13,14])$ :

$$
\begin{aligned}
F_{\alpha}\{f(x)\} & =f_{\omega}^{F, \alpha}(\omega) \\
& :=\frac{1}{\Gamma(1+\alpha)} \int_{-\infty}^{\infty} E_{\alpha}\left(-i^{\alpha} \omega^{\alpha} x^{\alpha}\right) f(x)(d x)^{\alpha} .
\end{aligned}
$$

Its inverse is formulated as follows (see $[13,14])$ :

$$
\begin{aligned}
f(x) & =F_{\alpha}^{-1}\left(f_{\omega}^{F, \alpha}(\omega)\right) \\
& :=\frac{1}{(2 \pi)^{\alpha}} \int_{-\infty}^{\infty} E_{\alpha}\left(i^{\alpha} \omega^{\alpha} x^{\alpha}\right) f_{\omega}^{F, \alpha}(\omega)(d \omega)^{\alpha}, \quad x>0 .
\end{aligned}
$$

Let $\varphi(x) \in L_{2, \alpha}[\mathbb{R}]$ and let

$$
\begin{array}{r}
\varphi_{\omega}^{F, \alpha}(\omega)=\frac{1}{\Gamma(1+\alpha)} \int_{-\infty}^{\infty} E_{\alpha}\left(-i^{\alpha} \omega^{\alpha} x^{\alpha}\right) \varphi(x)(d x)^{\alpha}, \\
0<\alpha \leq 1 .
\end{array}
$$

When

$$
\varphi_{\omega}^{F, \alpha}(\omega)=\frac{1}{\Gamma(1+\alpha)} \int_{-\infty}^{\infty} \varphi(x)(d x)^{\alpha}=0, \quad 0<\alpha \leq 1,
$$

the function $\varphi(x)$ is called a local fractional wavelet [13].

Let $\varphi(x) \in L_{2, \alpha}[\mathbb{R}]$. Then, we have

$$
\begin{aligned}
\left\|\varphi_{a, b, \alpha}(t)\right\|_{1, \alpha}^{2} & =\frac{1}{a^{\alpha} \Gamma(1+\alpha)} \int_{-\infty}^{\infty}\left|\varphi_{a, b, \alpha}(t)\right|^{2}(d x)^{\alpha} \\
& =\frac{1}{\Gamma(1+\alpha)} \int_{-\infty}^{\infty}|\varphi(t)|^{2}(d x)^{\alpha}=\|\varphi\|_{1, \alpha}^{2},
\end{aligned}
$$

so that

$$
\varphi_{a, b, \alpha}(t)=\frac{1}{a^{\alpha / 2}} \varphi\left(\frac{t-b}{a}\right),
$$

where $a, b \in \mathbb{R}$ and $a \neq 0$.

\section{Local Fractional Continuous Wavelet Transform}

Let $\varphi \in L_{2, \alpha}[\mathbb{R}]$. Then, we arrive at the following relation:

$$
\begin{aligned}
\left\|\varphi_{a, b, \alpha}(t)\right\|_{2, \alpha}^{1} & =\frac{1}{a^{\alpha} \Gamma(1+\alpha)} \int_{-\infty}^{\infty}\left|\varphi_{a, b, \alpha}(t)\right|^{2}(d t)^{\alpha} \\
& =\frac{1}{\Gamma(1+\alpha)} \int_{-\infty}^{\infty}|\varphi(t)|^{2}(d t)^{\alpha}=\|\varphi\|_{2, \alpha}^{1},
\end{aligned}
$$

where $\varphi_{a, b, \alpha}(t)=\left(1 / a^{\alpha / 2}\right) \varphi((t-b) / a), a, b \in \mathbb{R}$, and $a \neq 0$.

Similarly, we get

$$
\left\|\varphi_{b, \alpha}(t)\right\|_{2, \alpha}^{1}=\|\varphi\|_{2, \alpha}^{1} .
$$

Taking $\varphi_{b, \alpha}(t)$ in place of $\varphi_{a, b, \alpha}(t) E_{\alpha}\left(-i^{\alpha} \omega^{\alpha} t^{\alpha}\right)$, we obtain

$$
\begin{aligned}
\Theta_{\varphi_{a, b, \alpha}} f(a, b) & =\left\langle f(t), \varphi_{a, b, \alpha}(t)\right\rangle \\
& =\frac{1}{\Gamma(1+\alpha)} \int_{-\infty}^{\infty} f(t) \overline{\varphi_{a, b, \alpha}(t)}(d t)^{\alpha} .
\end{aligned}
$$

In the special case when $f(t)=1$, we have the following relation:

$$
\Theta_{\varphi_{a, b, \alpha}}(a, b)=\frac{1}{\Gamma(1+\alpha)} \int_{-\infty}^{\infty} \overline{\varphi_{a, b, \alpha}(t)}(d t)^{\alpha}
$$

such that

$$
\begin{gathered}
\frac{1}{\Gamma(1+\alpha)} \int_{-\infty}^{\infty} \Theta_{\varphi_{a, b, \alpha}}(a, b) \varphi_{a, b, \alpha}(t)(d b)^{\alpha} \\
=|a|^{\alpha}\left[\frac{1}{\Gamma(1+\alpha)} \int_{-\infty}^{\infty} \varphi(t)(d t)^{\alpha}\right]^{2} .
\end{gathered}
$$

Hence, there exists the following relation:

$$
\begin{aligned}
& \frac{1}{\Gamma^{2}(1+\alpha)} \iint_{-\infty}^{\infty} a^{-2 \alpha} \Theta_{\varphi_{a, b, \alpha}}(a, b) \varphi_{a, b, \alpha}(t)(d a)^{\alpha}(d b)^{\alpha} \\
& =\frac{1}{\Gamma(1+\alpha)} \int_{-\infty}^{\infty}|x|^{-\alpha}(d x)^{\alpha} .
\end{aligned}
$$


In general, we also deduce the following identities:

$$
\begin{aligned}
& f(x)= \frac{\int_{-\infty}^{\infty}\left(|f(x)|^{2} /|x|^{\alpha}\right)(d x)^{\alpha}}{\Gamma^{3}(1+\alpha)} \\
& \times \iint_{-\infty}^{\infty} a^{-2 \alpha} \Theta_{\varphi_{a, b, \alpha}} f(a, b) \varphi_{a, b, \alpha}(t)(d b)^{\alpha}(d a)^{\alpha}, \\
& \Theta_{\varphi_{a, b, \alpha}} f(a, b)=\frac{1}{\Gamma(1+\alpha)} \int_{-\infty}^{\infty} f(t) \overline{\varphi_{a, b, \alpha}(t)}(d t)^{\alpha} .
\end{aligned}
$$

Now, we establish the following relations:

$$
\begin{aligned}
\Theta_{\varphi_{a, b, \alpha} f(a, b)=} & \frac{a^{-\alpha / 2}}{\Gamma(1+\alpha)} \int_{-\infty}^{\infty} f(t) \overline{\varphi_{a, b, \alpha}(t)}(d t)^{\alpha}, \\
f(x)= & \frac{\int_{-\infty}^{\infty}\left(|f(x)|^{2} /|x|^{\alpha}\right)(d x)^{\alpha}}{\Gamma^{3}(1+\alpha)} \\
& \times \iint_{-\infty}^{\infty} a^{-2 \alpha} \Theta_{\varphi_{a, b, \alpha}} \widetilde{f(a, b)} \varphi_{a, b, \alpha} \\
& \times(t)(d a)^{\alpha}(d b)^{\alpha} .
\end{aligned}
$$

Hence, the local fractional continuous wavelet transform takes the following form (see [13]):

$$
W_{\varphi, \alpha} f(a, b)=\frac{a^{-\alpha / 2}}{\Gamma(1+\alpha)} \int_{-\infty}^{\infty} f(t) \overline{\varphi_{a, b, \alpha}(t)}(d t)^{\alpha}, \quad 0<\alpha \leq 1 .
$$

And the inversion formula of local fractional continuous wavelet transform is derived as follows (see [14]):

$$
\begin{aligned}
f(x)= & \frac{C_{\varphi, \alpha}}{\Gamma^{2}(1+\alpha)} \\
& \times \iint_{-\infty}^{\infty} a^{-2 \alpha} W_{\varphi, \alpha} f(a, b) \varphi_{a, b, \alpha}(t)(d a)^{\alpha}(d b)^{\alpha}, \\
& 0<\alpha \leq 1,
\end{aligned}
$$

where

$$
C_{\varphi, \alpha}=\frac{1}{\Gamma(1+\alpha)} \int_{-\infty}^{\infty} \frac{|f(x)|^{2}}{|x|^{\alpha}}(d x)^{\alpha}, \quad 0<\alpha \leq 1 .
$$

\section{The Wavelet Space}

In order to differ the classical wavelets from fractional wavelets, here we formulate a wavelet space as follows. In fact, a wavelet space is defined by

$$
\begin{aligned}
W_{\varphi, \alpha}[\mathbb{R}]=\left\{(\varphi, \alpha): W_{\varphi, \alpha} f(a, b)\right. \\
\left.\quad=\frac{a^{-\alpha / 2}}{\Gamma(1+\alpha)} \int_{-\infty}^{\infty} f(t) \overline{\varphi_{a, b, \alpha}(t)}(d t)^{\alpha}, 0<\alpha \leq 1\right\} .
\end{aligned}
$$

When the fractal dimension $\alpha$ is equal to 1 , from (27), we deduce (see [3-5])

$$
\begin{aligned}
W_{\varphi, 1}[\mathbb{R}]=\left\{(\varphi, 1): W_{\varphi, 1} f(a, b)\right. \\
\left.=a^{-1 / 2} \int_{-\infty}^{\infty} f(t) \overline{\varphi_{a, b, 1}(t)} d t, \alpha=1\right\},
\end{aligned}
$$

where $f(t)$ is continuous and $W_{\varphi, 1} f(a, b) \in W_{\varphi, 1}[\mathbb{R}]$.

Taking the fractal dimension $0<\alpha<1$, we derive a formula given by

$$
W_{\varphi, \alpha} f(a, b)=\frac{a^{-\alpha / 2}}{\Gamma(1+\alpha)} \int_{-\infty}^{\infty} f(t) \overline{\varphi_{a, b, \alpha}(t)}(d t)^{\alpha}
$$

with $W_{\varphi, \alpha} f(a, b) \in W_{\varphi, \alpha}[\mathbb{R}]$, where $f(t)$ is a local fractional continuous function.

\section{An Illustrative Example}

In order to construct the local fractional continuous wavelet, we suppose that $\phi(t)$ is $m \alpha$ times the local fractional differentiable function.

We define the local fractional wavelet $\varphi(t)$ by means of the following expression:

$$
\varphi(t)=\frac{d^{m \alpha} y}{d x^{m \alpha}}
$$

where the differential operator is the local fractional operator proposed by Yang [18] (for other definition, see [19] and the references cited therein).

Then, we get

$$
\begin{aligned}
& \frac{1}{\Gamma(1+\alpha)} \int_{-\infty}^{\infty} \varphi(t) \frac{t^{m \alpha}}{\Gamma(1+m \alpha)}(d t)^{\alpha} \\
& =0 \quad(m=0,1,2, \ldots, m) .
\end{aligned}
$$

Let us consider the following nondifferentiable signal, namely,

$$
\phi_{H(\alpha)}(t)= \begin{cases}\frac{t^{\alpha}}{\Gamma(1+\alpha)}, & 0 \leq t<\frac{1}{2}, \\ \frac{(1-t)^{\alpha}}{\Gamma(1+\alpha)}, & \frac{1}{2} \leq t<1, \\ 0, & \text { else. }\end{cases}
$$

For $0 \leq t<1 / 2$, we obtain

$$
\frac{d^{\alpha} \phi_{H(\alpha)}(t)}{d t^{\alpha}}=\frac{d^{\alpha}}{d t^{\alpha}} \frac{t^{\alpha}}{\Gamma(1+\alpha)}=1 .
$$

For $1 / 2 \leq t<1$, we obtain

$$
\frac{d^{\alpha} \phi_{H(\alpha)}(t)}{d t^{\alpha}}=\frac{d^{\alpha}}{d t^{\alpha}} \frac{(1-t)^{\alpha}}{\Gamma(1+\alpha)}=-1 .
$$


In view of (33)-(34), we get a local fractional wavelet given by

$$
\varphi_{H(\alpha)}(t)= \begin{cases}1, & 0 \leq t<\frac{1}{2}, \\ -1, & \frac{1}{2} \leq t<1, \\ 0, & \text { else. }\end{cases}
$$

Following (35), we obtain

$$
\begin{aligned}
& \frac{1}{\Gamma(1+\alpha)} \int_{-\infty}^{\infty} \varphi_{H(\alpha)}(t)(d t)^{\alpha}=0, \\
& \frac{1}{\Gamma(1+\alpha)} \int_{-\infty}^{\infty} \varphi_{H(\alpha)}^{2}(t)(d t)^{\alpha}=1 .
\end{aligned}
$$

In view of (15), taking $a=2^{-j}$ and $b=k 2^{-j}$, we have

$$
\begin{aligned}
\varphi_{a, b, \alpha}(t) & =\frac{1}{a^{\alpha / 2}} \varphi\left(\frac{t-b}{a}\right)=\varphi_{j, k, \alpha}(t) \\
& =\varphi_{2^{-j}, k 2^{-j}, \alpha}(t)=2^{j \alpha / 2} \varphi\left(2^{j} t-k\right)
\end{aligned}
$$

for integers $j, k \in \mathbb{Z}$.

Hence, we get the following equation:

$$
\varphi_{H(\alpha)}^{j, k}(t)=2^{j \alpha / 2} \varphi_{H(\alpha)}\left(2^{j} t-k\right)
$$

We thus conclude that

$$
\begin{aligned}
& \frac{1}{\Gamma(1+\alpha)} \int_{-\infty}^{\infty}\left[\varphi_{H(\alpha)}^{j, k}(t)\right]^{2}(d t)^{\alpha} \\
& =\frac{1}{\Gamma(1+\alpha)} \int_{-\infty}^{\infty}\left[2^{j \alpha / 2} \varphi_{H(\alpha)}\left(2^{j} t-k\right)\right]^{2}(d t)^{\alpha} \\
& =2^{j \alpha} \frac{1}{\Gamma(1+\alpha)} \int_{-\infty}^{\infty}\left[\varphi_{H(\alpha)}\left(2^{j} t-k\right)\right]^{2}(d t)^{\alpha} \\
& =\frac{1}{\Gamma(1+\alpha)} \int_{-\infty}^{\infty}\left[\varphi_{H(\alpha)}\left(2^{j} t-k\right)\right]^{2}\left(d\left(2^{j} t-k\right)\right)^{\alpha} \\
& =\frac{1}{\Gamma(1+\alpha)} \int_{-\infty}^{\infty}\left[\varphi_{H(\alpha)}(t)\right]^{2}(d t)^{\alpha} \\
& =1 \text {, } \\
& \frac{1}{\Gamma(1+\alpha)} \int_{-\infty}^{\infty} \varphi_{H(\alpha)}^{j, k}(t)(d t)^{\alpha} \\
& =\frac{1}{\Gamma(1+\alpha)} \int_{0}^{1 / 2} 2^{j \alpha / 2} \varphi_{H(\alpha)}\left(2^{j} t-k\right)(d t)^{\alpha} \\
& -\frac{1}{\Gamma(1+\alpha)} \int_{1 / 2}^{1} 2^{j \alpha / 2} \varphi_{H(\alpha)}\left(2^{j} t-k\right)(d t)^{\alpha} \\
& =0 \text {. }
\end{aligned}
$$

\section{Concluding Remarks and Observations}

A novel local fractional wavelet transformation was investigated by using Fourier transform based upon local fractional calculus. This transform has been found to be advantageous in dealing with the functions in fractal space. The wave space is considered and an illustrative example is shown.

\section{Conflict of Interests}

The authors declare that they have no conflict of interests regarding this paper.

\section{References}

[1] I. Daubechies, "The wavelet transform, time-frequency localization and signal analysis," IEEE Transactions on Information Theory, vol. 36, no. 5, pp. 961-1005, 1990.

[2] R. K. Martinet, J. Morlet, and A. Grossmann, "Analysis of sound patterns through wavelet transforms," Journal of Pattern Recognition and Artificial Intelligence, vol. 1, no. 2, pp. 273-302, 1987.

[3] C. K. Chui, An Introduction to Wavelets, Academic Press, San Diego, Calif, USA, 1992.

[4] L. Debnath, Wavelet Transforms and Their Application, Birkhäuser, Boston, Mass, USA, 2002.

[5] C. Cattani, "Harmonic wavelet solution of Poisson's problem," Balkan Journal of Geometry and its Applications, vol. 13, no. 1, pp. 27-37, 2008.

[6] C. Cattani and J. Rushchitsky, Wavelet and Wave Analysis as Applied to Materials with Micro or Nanostructure, World Scientific, 2007.

[7] D. Mendlovic, Z. Zalevsky, D. Mas, J. García, and C. Ferreira, "Fractional wavelet transform," Applied Optics, vol. 36, no. 20, pp. 4801-4806, 1997.

[8] L. Chen and D. Zhao, "Optical image encryption based on fractional wavelet transform," Optics Communications, vol. 254, no. 4-6, pp. 361-367, 2005.

[9] E. Dinç, F. Demirkaya, D. Baleanu, Y. Kadioğlu, and E. Kadioğlu, "New approach for simultaneous spectral analysis of a complex mixture using the fractional wavelet transform," Communications in Nonlinear Science and Numerical Simulation, vol. 15, no. 4, pp. 812-818, 2010.

[10] E. Dinç and D. Baleanu, "Fractional wavelet transform for the quantitative spectral resolution of the composite signals of the active compounds in a two-component mixture," Computers \& Mathematics with Applications, vol. 59, no. 5, pp. 1701-1708, 2010.

[11] E. Dinç, D. Baleanu, and K. Taş, "Fractional wavelet analysis of the composite signals of two-component mixture by multivariate spectral calibration," Journal of Vibration and Control, vol. 13, no. 9-10, pp. 1283-1290, 2007.

[12] J. Shi, N. Zhang, and X. Liu, "A novel fractional wavelet transform and its applications," Science China Information Sciences, vol. 55, no. 6, pp. 1270-1279, 2012.

[13] X. J. Yang, Local Fractional Functional Analysis and Its Applications, Asian Academic publisher, Hong Kong, China, 2011.

[14] X. J. Yang, D. Baleanu, and J. T. A. Machado, "Mathematical aspects of Heisenberg uncertainty principle within local fractional Fourier analysis," Boundary Value Problems, vol. 2013, no. 1, article 131, 2013.

[15] X. J. Yang and D. Baleanu, "Fractal heat conduction problem solved by local fractional variation iteration method," Thermal Science, vol. 17, no. 2, pp. 625-628, 2013.

[16] A.-M. Yang, X.-J. Yang, and Z.-B. Li, "Local fractional series expansion method for solving wave and diffusion equations on Cantor sets," Abstract and Applied Analysis, vol. 2013, Article ID 351057, 5 pages, 2013. 
[17] W.-H. Su, D. Baleanu, X.-J. Yang, and H. Jafari, "Damped wave equation and dissipative wave equation in fractal strings within the local fractional variational iteration method," Fixed Point Theory and Applications, vol. 2013, article 89, 2013.

[18] X. J. Yang, Advanced Local Fractional Calculus and Its Applications, World Science, New York, NY, USA, 2012.

[19] A. A. Kilbas, H. M. Srivastava, and J. J. Trujillo, Theory and Applications of Fractional Differential Equations, vol. 204, Elsevier Science, Amsterdam, The Netherlands, 2006. 


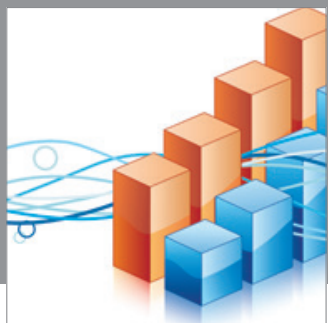

Advances in

Operations Research

mansans

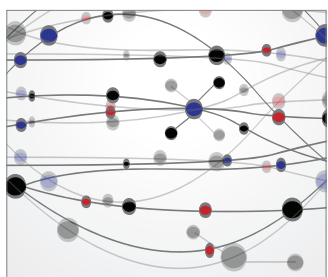

The Scientific World Journal
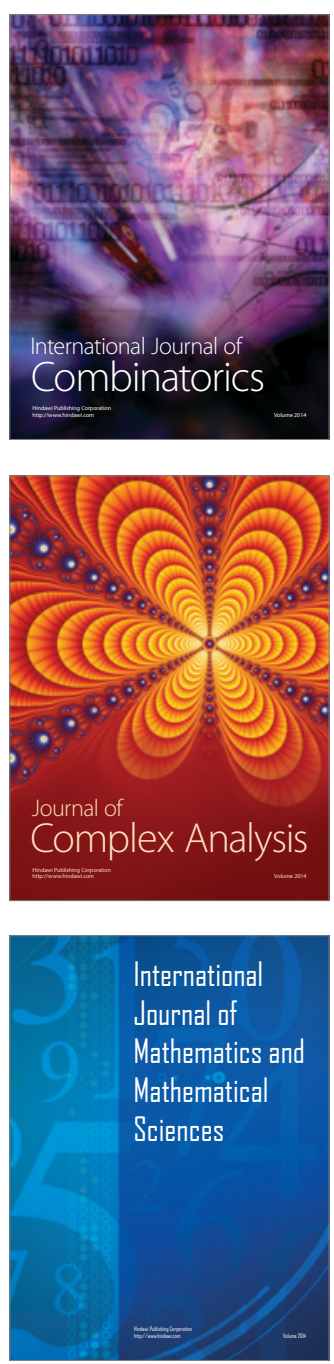
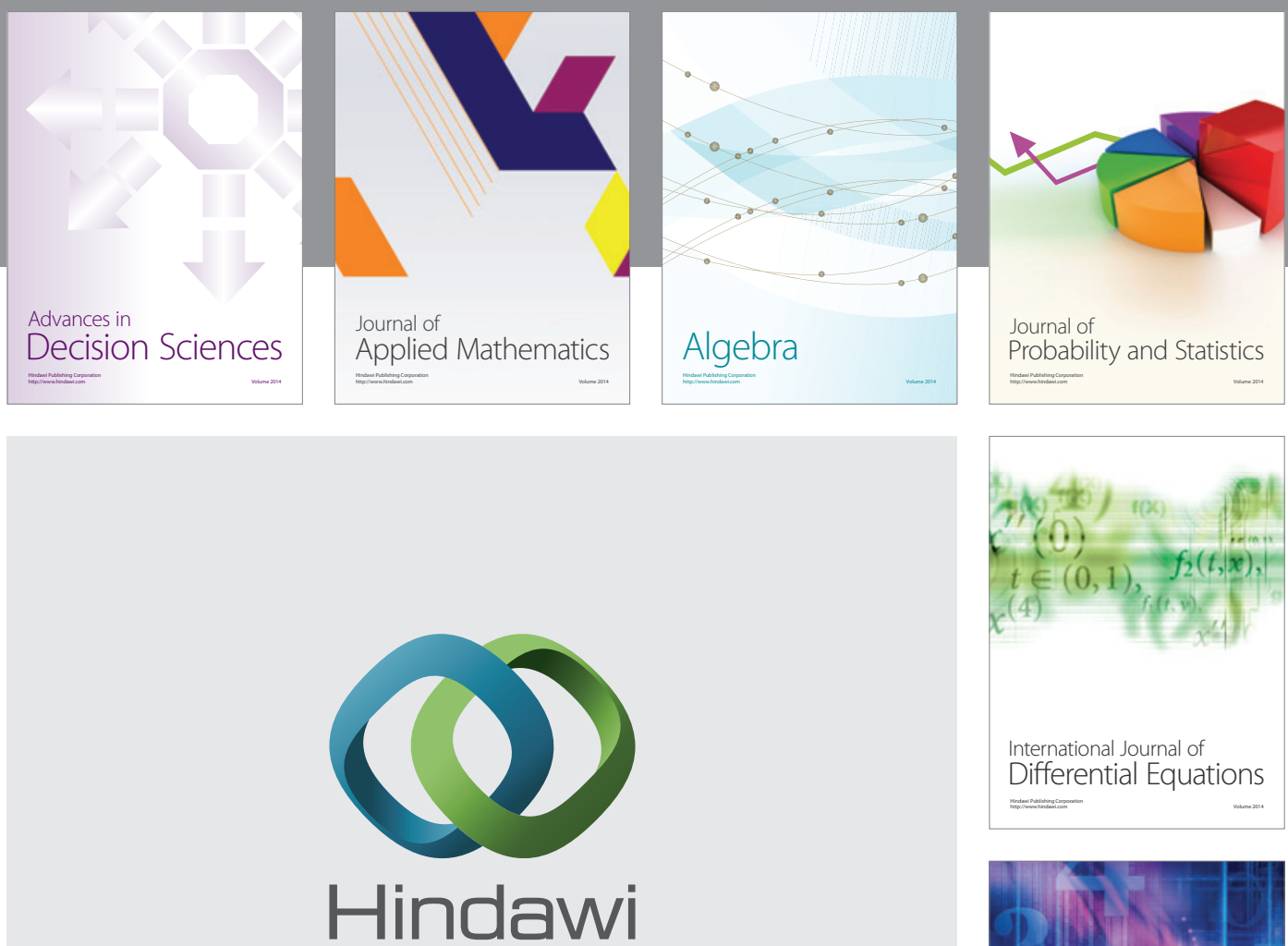

Submit your manuscripts at http://www.hindawi.com
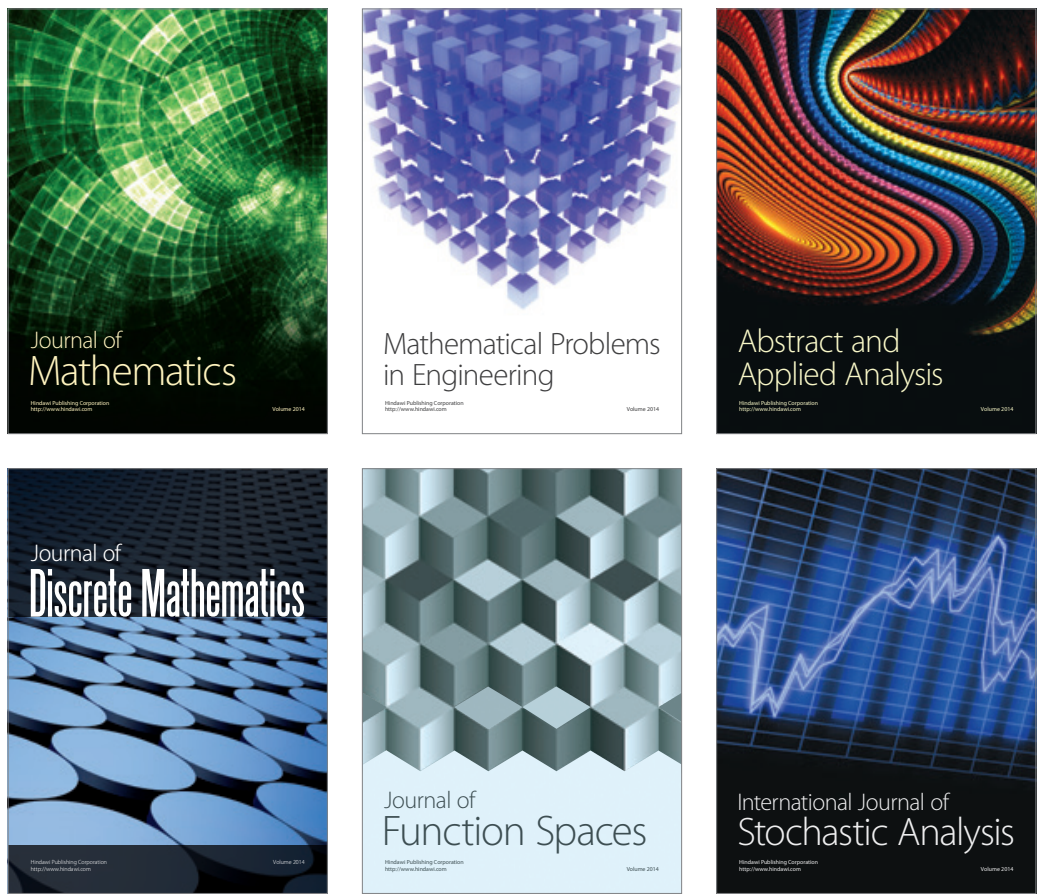

Journal of

Function Spaces

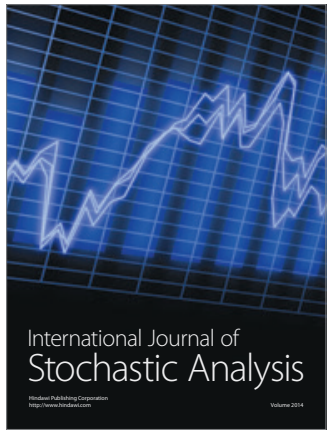

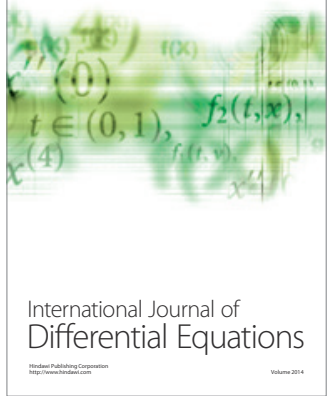
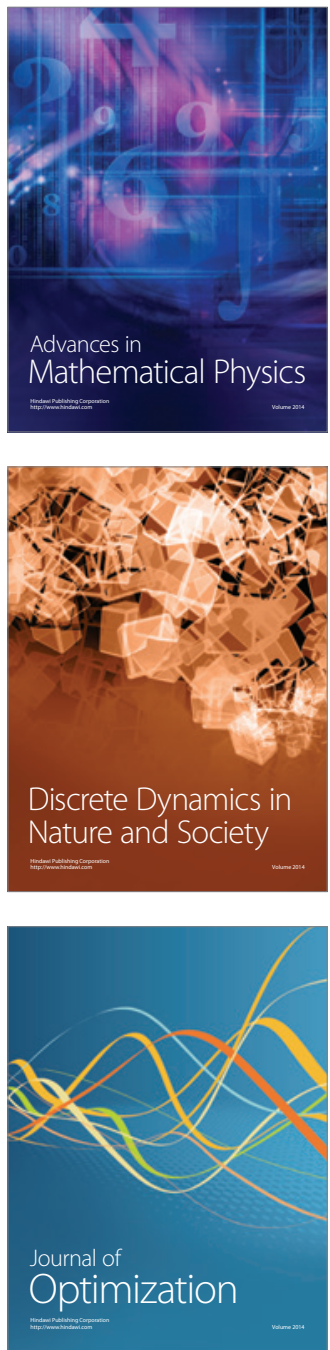